\title{
Research on Flow-induced Vibration Characteristics of Conveying Pipes Under Bidirectional Tidal Flow
}

\author{
W Chunsheng, L Zejun, Z Yan, S Qiji* \\ Department of Petroleum Engineering, Northeast \\ Petroleum University, Heilongjiang Daqing 163318, China
}

\begin{abstract}
Pipeline-induced vibration phenomena are caused by waves and currents during the transportation of oil and natural gas pipelines. For this purpose, the multiphysics coupling calculation was employed by considering the influence of fluid flow characteristics in ocean tidal flow environment and mechanical properties of pipeline solid structure on vibration characteristics of S-type pipeline. According to the analysis of the velocity of the fluid domain and the distribution of the stress distribution of the pipeline, combined with the time history and amplitude of the monitoring point and axis of the pipeline, we analyzed the flow-induced vibration response characteristics of the S-transport pipeline. The results show that under the cyclic flow of the two-way tidal basin, the velocity field on the deformation side of the pipeline is lower, the stress at the solid support end is higher than that in the riser region, the time-displacement of the pipeline fluctuates periodically, and the displacement of the pipeline axis increases sharply in the riser pipe section. It exhibits a normal distribution with extreme values at the center of the pipe segment. The maximum single-pass amplitude in the verticality basin is twice that of the parallel basin and the maximum amplitude is about $1 \mathrm{D}$.
\end{abstract}

\section{INTRODUCTION}

The pipelines that are put into operation in the sea area are subject to eddy vibrations caused by the turbulence of the two-way tidal stream, resulting in excessive fatigue, reduced life, small cracks and even damage [1-2]. Therefore, the safety of pipeline system operation has always been the focus of attention.

In recent years, some scholars have begun to pay attention to the vibration of pipelines under the action of internal and external flow excitation. Meng Dan, Guo Haiyan et al. [3] considered the combined effect of internal and external fluids to study the fluid-induced vibration stability of the pipeline. Bao Ridong, Liang Feng et al. [4] predicted the bifurcation and chaotic motion behaviors of the end-constrained cantilever transport pipeline, the fixedconveying pipeline at both ends and the general supporting transport pipeline and determined the basis. Chica et al. [5] proposed a fluid-structure coupling method combining computational fluid dynamics (CFD) and computational structural mechanics (CSD). Using this method, two-way fluid-structure coupling calculations were performed on the two elbows of the Mtype underwater rigid bridge.

$\overline{{ }^{*} \text { Corresponding Author: sunqiji@nepu.edu.cn }}$ 
The CFD calculation of the entire model was carried out. The volume fraction of multiphase flow in the pipeline was obtained through simulation. The frequency of the slug flow in the pipeline was obtained by tracing the slug. At the same time, the crossover pipe under the action of multiphase flow was obtained. Dynamic response and modal analysis of the crossover tube based on this. D. Jia [6] applied a similar method to predict the formation and development of the slug flow of underwater straight pipes, bent pipes and crossover pipes, and obtained the pressure distribution of the flow field and the stress distribution of the pipeline under the action of multiphase flow. Juan et al. [7] simulated the flow-induced vibration phenomenon caused by the multiphase flow internal flow of the crossover pipe of the underwater straight pipe joint. CFD technique shows good potential in FIV research [8$10]$. Based on this background, this study is carried out.

Another noteworthy problem is that the fluid-solid coupling vibration problem of the transport pipeline is a typical infinite-dimensional continuous gyroscopic system dynamics model. Bourguet et al. [11-13] studied the vortex-induced vibration of a flexible riser in shear flow with different Reynolds numbers. It was found that the cylindrical vibration contains both mixed and standing wave vibration modes, and at different directions along the riser are respectively a certain frequency prone to vortex splitting between the vortex regions. Song Leijian et al. [14-15] used the model test method to study the vortex-induced vibration (VIV) response characteristics and vortex force load characteristics of a flexible riser under uniform flow and analyzed the VIV response characteristics of the flexible riser in a uniform flow field. Chun Ning et al. [16-17] studied the flow-induced vibration of tandem double cylinders, parallel double cylinders and slender flexible cylinders.

Based on the rigid cylindrical vortex-induced vibration of elastic support, combined with the flow dynamics equation and the mechanical equation of pipeline solid structure, the fluidsolid coupling calculation was carried out to fluidize the pipeline vibration caused by the flow of the two-way tidal flow in the S-transport pipeline. According to the coupling calculation and analysis, we calculated the velocity field changes caused by fluid periodic motion in the parallel and verticality basins and the stress and displacement of the pipeline wall, and then analyzed the periodic deformation and vibration characteristics of the pipeline.

\section{PHYSICAL MODEL}

With the development of marine oil and gas exploration technology, flexible risers are often used in marine engineering. Considering the flow-induced vibration phenomenon of the large number of catenary-type pipelines in the offshore oil and gas project affected by the large ocean current and the aspect ratio of the pipeline, the S-type flow pipeline with the inlet and outlet support is in the tidal basin of parallel and verticality bidirectional periodic motion. In this paper, we mainly studied the velocity field changes caused by fluid periodic motion in the parallel and verticality basins, as well as the stress and displacement of the pipeline wall, and analyzed the periodic deformation and vibration characteristics of the pipeline. The flowinduced vibration characteristics of the basin in the pipeline were ignored.

The schematic diagram of the physical model of the S-transport pipeline in the two-way tidal basin is shown in Fig 1(b).The monitoring points A1, B1, C1, D1 and E1 were set in the riser area and the elbow area respectively, and the time-lapse displacement of the riser in the basin was detected. Detailed piping system parameters and fluid property parameters are shown in Tab 1. 

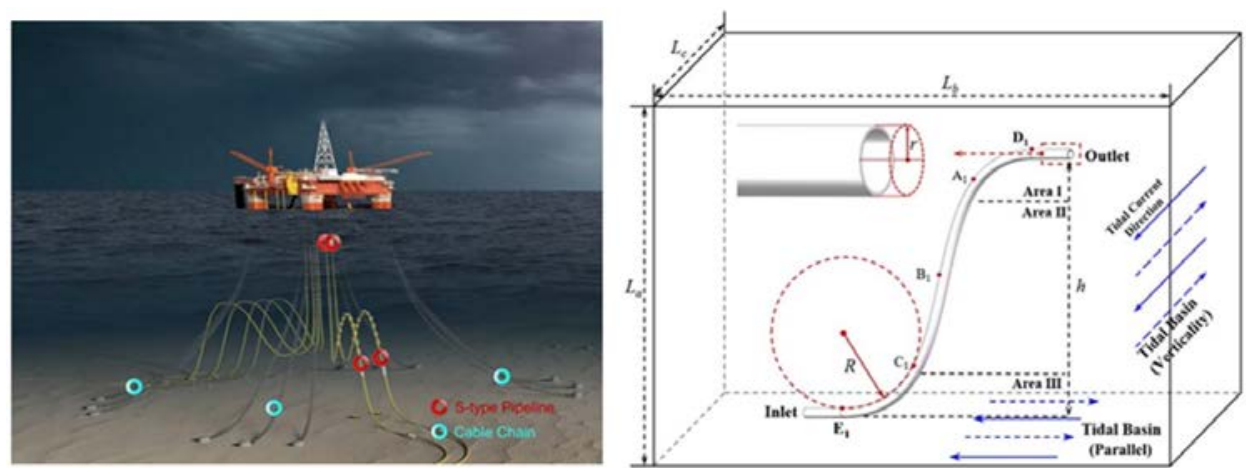

Figure 1: Physical model of S-transport pipeline in two-way tidal basin

Table 1S-transport pipeline parameters in two-way tidal basin

\begin{tabular}{llcllc}
\hline Model & $\begin{array}{l}\text { Physical } \\
\text { Parameters }\end{array}$ & Value & Model & \multicolumn{2}{l}{ Basin Parameters Value } \\
\hline S-type & Length [m] & 30 & Outflow Basin & $\begin{array}{l}\text { Fluid density } \\
{\left[\mathrm{kg} / \mathrm{m}^{3}\right]}\end{array}$ & 1013 \\
Pipeline & & & & Flow rate $[\mathrm{m} / \mathrm{s}]$ & $\leq 4$ \\
& Outer Diameter [m] & 0.6 & & Flow direction & Parallel \\
& Inside & 0.55 & & \\
& Diameter [m] & & & Verticality \\
& Wall Thickness [m] & 0.025 & X direction [m] & 20 \\
& Density [kg/m $\left.{ }^{3}\right]$ & 7850 & Y direction [m] & 20 \\
& Young's & $2 \times 1011$ & & \\
& Modulus [Pa] & & Z direction [m] & 20 \\
\hline
\end{tabular}

\section{MULTIPHYSICS COUPLING NUMERICAL METHOD FOR PIPELINE}

The governing equations of fluid flow studied in this paper are the incompressible NavierStokes equations (Equation 1) described by ALE. A four-step split finite element method was used for the time and space discretization; the structural dynamics equations (Equations 7 and 8) were solved by the method of explicit direct integration, the update of the fluid calculation grid was realized by the Laplace method, and the mutual coupling between the fluid and the structure adopted the weakly coupled partition algorithm. COMSOL Multiphysics 5.3a platform was based on the partial differential multi-physics coupled numerical method for different specific problems, static and dynamic analysis, linear and nonlinear analysis, and eigenvalue and modal analysis.

\subsection{Fluid Control Equation}

Continuity equation is given in equation (1):

$$
\frac{\delta \rho_{t}}{\delta t}+\nabla g\left(\rho_{t} U_{\text {fluid }}\right)=0
$$


where $\rho_{t}$ is the fluid density $\rho t$ is time, $U_{\text {fluid }}$ is the speed vector. Due to the assumption that the fluid is incompressible during the study, the density is constant, and so $\frac{\delta \rho_{t}}{\delta t}=0$, equation (1) can be written as equation (2):

$$
\nabla \mathrm{g}\left(\rho_{t} U_{\text {fluid }}\right)=0
$$

Equation of motion: According to the law of conservation of motion, the basic form of the equation of motion can be written as equation (3):

$$
\rho_{t} \frac{d U_{\text {fluid }}}{d t}=\nabla \mathrm{g} \sigma+\rho_{t} \mathrm{~g}
$$

The gravity of the fluid is ignored in this study, and then equation (3) can be written as equation (4):

$$
\rho_{t} \frac{d U_{\text {fluid }}}{d t}-\nabla \mathrm{g \sigma}=0
$$

Stress tensor can be decomposed into equation (5):

$$
\sigma=-p I+\tau
$$

where $p$ is the static pressure, $I$ is the unit matrix and $\tau$ is the deviatoric stress tensor. Then (5) can be written as shown in equation (6):

$$
\rho_{t} \frac{d U_{\text {fluid }}}{d t}=\nabla g \sigma-\nabla p
$$

\subsection{Structural Control Equation}

Structural dynamics governing equations are shown as follows in equation (7) and (8):

$$
\begin{aligned}
& m \frac{d^{2} x}{d t^{2}}+c \frac{d x}{d t}+k x=F_{x}(t) \\
& m \frac{d^{2} y}{d t^{2}}+c \frac{d y}{d t}+k y=F_{y}(t)
\end{aligned}
$$

where $m$ is the mass of the cylinder, $c$ is the damping coefficient, and $k$ is the structural stiffness coefficient; $F_{x}(t), F_{y}(t)$ are respectively the forward and cross flow to the physical force, $F_{x}=\int_{\Gamma_{\text {wall }}} \sigma n_{x} d \Gamma, F_{y}=\int_{\Gamma_{\text {wall }}} \sigma n_{y} d \Gamma$ and $\sigma$ is the fluid stress tensor.

$$
\begin{gathered}
u_{\text {fluid }}=u_{w} \\
u_{w}=\frac{\partial u_{\text {fluid }}}{\partial t} \\
\sigma \cdot n=\Gamma \cdot \mathrm{n}, \Gamma=\left[-P I+\mu\left(\nabla u_{\text {fluid }}+\left(\nabla u_{\text {fluid }}\right)^{T}\right)-\frac{2}{3} \mu\left(\nabla \cdot \mu_{\text {fluid }}\right) I\right]
\end{gathered}
$$




\subsection{Two-way Tidal Basin Establishment}

For the outer basin where the S-type transport pipeline is located, there is a flow parallel to the direction of the transport pipeline and a flow perpendicular to the direction of the transport conduit (the flow-induced vibration induced by the tidal flow in the remaining directions is in the two flow directions studied in this paper). Therefore, a rectangular basin around the S-type transport pipeline was established, and the inside of the rectangular basin was defined as the tidal flow satisfying the Equation (10):

$$
U_{\text {fluid }}=4 \cdot \sin (\text { omega } \cdot t+0.01)
$$

where $U_{\text {fluid }}$ is the fluid flow rate for the outer basin, omega is the angular frequency, which is $0.2 \mathrm{~Hz}$ as shown in Equation (11),

$$
U_{\text {fluid }}=4 \cdot \sin (\text { omega } \cdot t+0.01)
$$

where $f$ is the velocity field frequency of the tidal basin, which is $1.256 \mathrm{~Hz}$.

\section{CALCULATION RESULTS AND ANALYSIS}

The numerical analysis of the vibration of the pipeline in the two-way tidal basin was carried out. Combined with the finite element solution of partial differential, the multi-physics coupling of the linear elastic equation and the fluid dynamics in solid mechanics was solved. Then the parallel basin and the verticality basin were calculated and analyzed. Meanwhile, we investigated the velocity field caused by the periodic motion of the fluid and the stress and displacement of the pipe wall of the pipeline and explored the periodic deformation and vibration characteristics of the pipeline.

\subsection{Flow-induced vibration analysis of bidirectional tidal flow}

Fig. 2 depicts the 10s basin velocity field under tidal flow conditions in the parallel and verticality basins. According to the speed nephogram display:

When the S-type flow pipeline exists in the parallel basin (ignoring the flow velocity change caused at the corner of the basin), the fluid around the S-type transport pipeline is obviously surrounded by the fluid-solid coupling effect, the flow velocity in the two solid supports of the transport pipeline vicinity is significantly larger, and the deformation of the pipe portion causes a difference in the surrounding flow field due to the deformation flutter. For the cross-sectional calculation of the basin, the fluid-solid coupling velocity field nephograms describing the $x-y$ plane, the $x-z$ plane and the $y-z$ plane of the transport pipeline were respectively obtained. It can be seen from the three-section nephogram of the $x-y$ plane that, except for the similar velocity field distribution at the entrance plane, the riser pipe section plane and the exit plane of the pipeline, the more uniform velocity field is divided by the pipe body in the rectangular basin. The velocity field around the pipe body is slightly higher; the velocity profile nephogram shown by the y-z plane shows that the velocity around the nozzle is significantly higher, which is related to the fixed connection of the pipe entrance and exit, and the stress is relatively large; It can be seen from the $\mathrm{x}-\mathrm{z}$ plane that the velocity field of the basin is divided by the pipe body into a trapezoidal distribution, and the velocity around the pipe body is large. 
When the S-type flow conduit exists in the verticality basin, the fluid flow in the basin is the tidal flow in the direction of the vertical pipeline axis, and the flow velocity change at the corners of the rectangular basin is ignored as in the parallel basin. The cut surface calculation was carried out for the basin. It can be seen that the tidal flow direction is the vertical pipe axis outward in the 10s time period. The outward velocity field distribution trend in different directions of the pipeline, and the contact between the basin and the fixed branch end of the pipeline have more obvious large velocity. Besides, the velocity field of the verticality basin changes with the change of the physical shape of the pipeline, and the fluid-solid coupling occurs between the basin and the pipeline. The fluid flow momentum is transmitted to the pipeline to deform it, and the basin is deformed with the deformation of the pipeline. The velocity field also changes with the deformation trend of the pipeline, that is, the fluid tidal flow in the basin induces the deformation flutter of the pipeline, and the deformation of the pipeline causes the velocity field change.

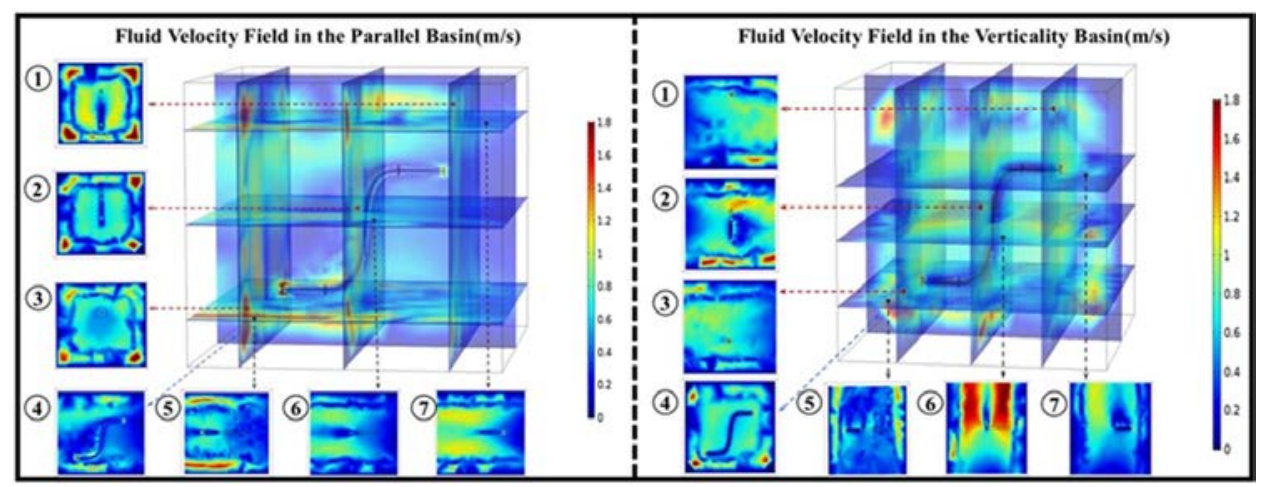

Figure 2: Velocity field distribution map in Parallel and Verticality Basins (1) (2)(3) represent the $X-Y$ section speed nephogram; (4) represents the $Y-Z$ section speed nephogram; (5) (6) (7) represent the $X-Z$ section speed nephogram)

\subsection{Stress field distribution of bidirectional tidal flow}

Combining the velocity field of the corresponding time domain, by calculating the stress caused by the Parallel basin on the S-type pipeline, it is known that the stress generated by the impact of the fluid on the pipeline in the basin is the direct cause of the fluttering of the pipeline deformation. The velocity of the nearby flow field is significantly lower than that of other areas in the basin. At the same time, the different stress values of the pipeline and the velocity fields near different deformation zones are different, which indicates that the physical properties of the tidal stream indirectly affect the occurrence and strength of the deformation. The pipe stress at different times within 10s is shown in Fig.3. It can be seen from the Fig.3 that the S-type flow pipeline has different displacements of different positions at different times in the Parallel flow field, that is, vibration occurs, and the displacement direction of the $1.3 \mathrm{~s}$ pipeline is lower than the initial position. The displacement direction of the $3.7 \mathrm{~s}$ is upward, and the 5.7s and 8.3s are both at the moment of weak displacement; at the same time, the velocity field corresponding to the time course shows that the velocity field in the displacement direction of the pipeline is lower, which is related to the pipeline stress caused by the momentum described above. 
The analysis that causes the vibration to occur is consistent; and the portion of the pipe with weaker displacement and no displacement is significantly higher than the area of the pipe where the displacement is more pronounced.

By calculating the stress and deformation of the pipeline in the verticality basin, it is known that the pipeline undergoes the same deformation flutter in the full cycle time course as the tidal flow period, in which the stress at the fixed end of the pipeline is higher than other regions. Therefore, the deformation near the solid support end is weak, the stress of the curved pipe is low, and the stress of the central part of the riser is relatively high, so that there is almost no staggered deformation of the riser pipe in the overall deformation of the riser pipe area.

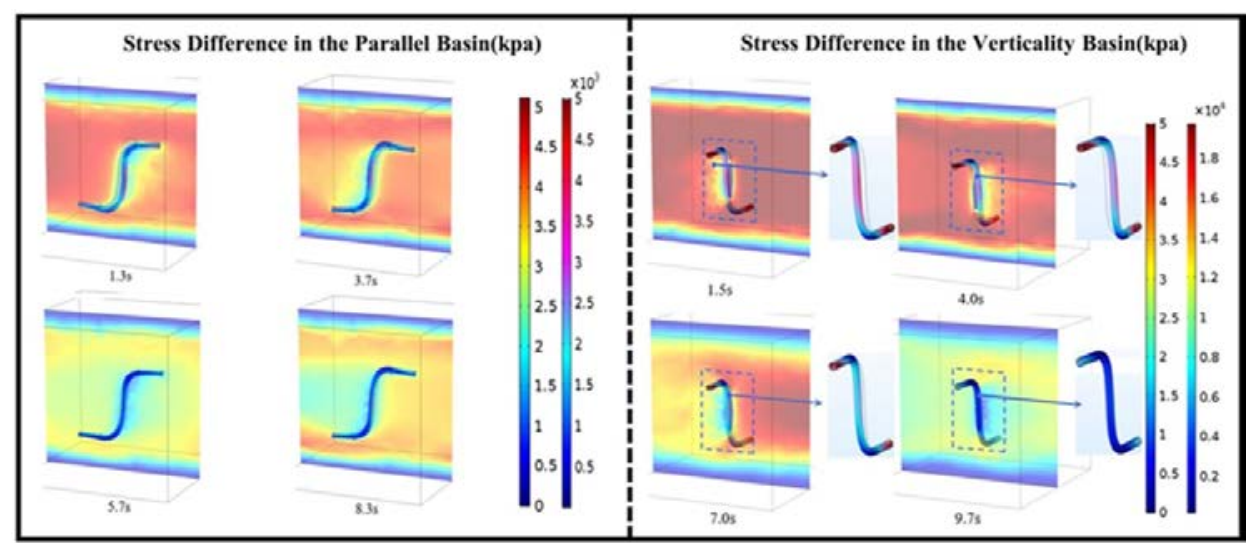

Figure 3: Fluctuation of stress field in pipelines of Parallel and Verticality Basin

\subsection{Dynamic tracking of flutter in bidirectional tidal flow}

The time-history displacement curve for the five monitoring points set in Fig. 1(b) (Fig. 4(a)) was calculated. The monitoring point B1 in the riser pipe section area has the largest timehistory displacement, and the turning point is closer to the entrance. The turn monitoring point $\mathrm{C} 1$ closer to the entrance and the turn monitoring point A1 closer to the exit are the second echelon. Besides, the monitoring point C1 is slightly larger, the time-history displacement of the monitoring point at the entrance and exit is the smallest, and all the monitoring points maintain the same fluctuation law and vibration frequency. The maximum amplitude is 0.40 $\mathrm{m}$ and the minimum amplitude is $0.120 \mathrm{~m}$. According to the calculation of the time-history displacement of the axis of the conveying pipe (Fig. 4(b)), the time-history displacement of the pipe axis is suddenly increased when the $x$-direction coordinate is 0 , and corresponds to the position of the three-dimensional pipeline in the parallel basin, but corresponds to the riser area of the flow pipe in the vicinity of the $x$ value of 0 ; the value of $x$ between -1 and 1 is the area between the turn of the inlet and outlet of the flow pipe. Thus, when the parallel basin fluid acts on the stress of the pipeline, deformation flutter is induced to the flow pipe, the riser pipe section becomes severely deformed, the deformation of the riser pipe toward the inlet and outlet ends is gradually weakened, and the time-displacement curve of the axis shows frequent chattering through the axis. 


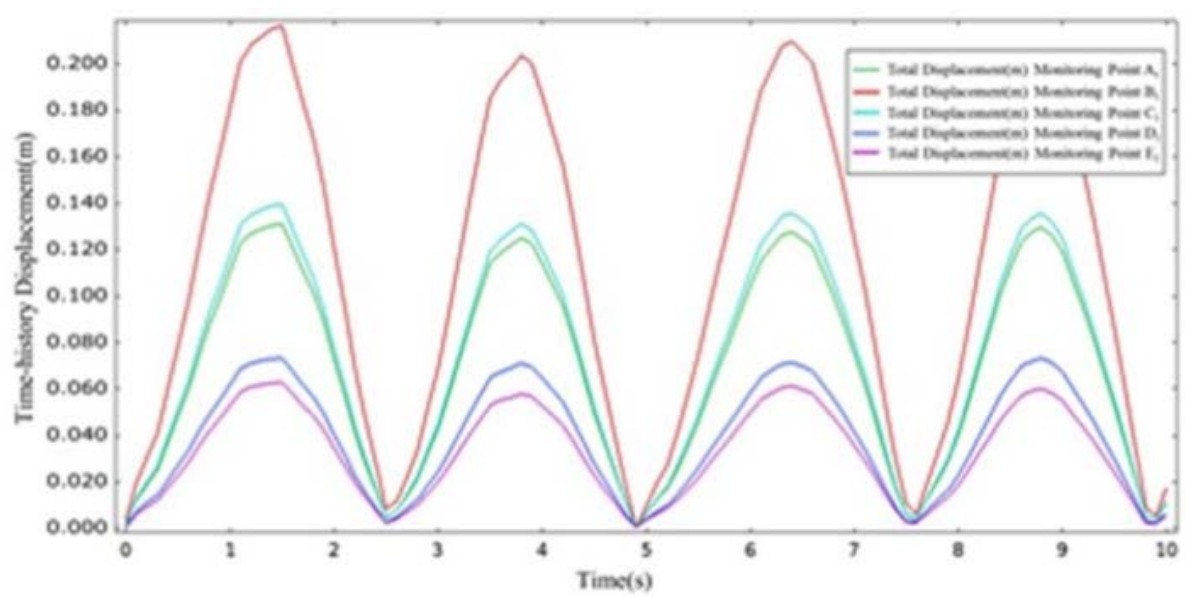

(a)

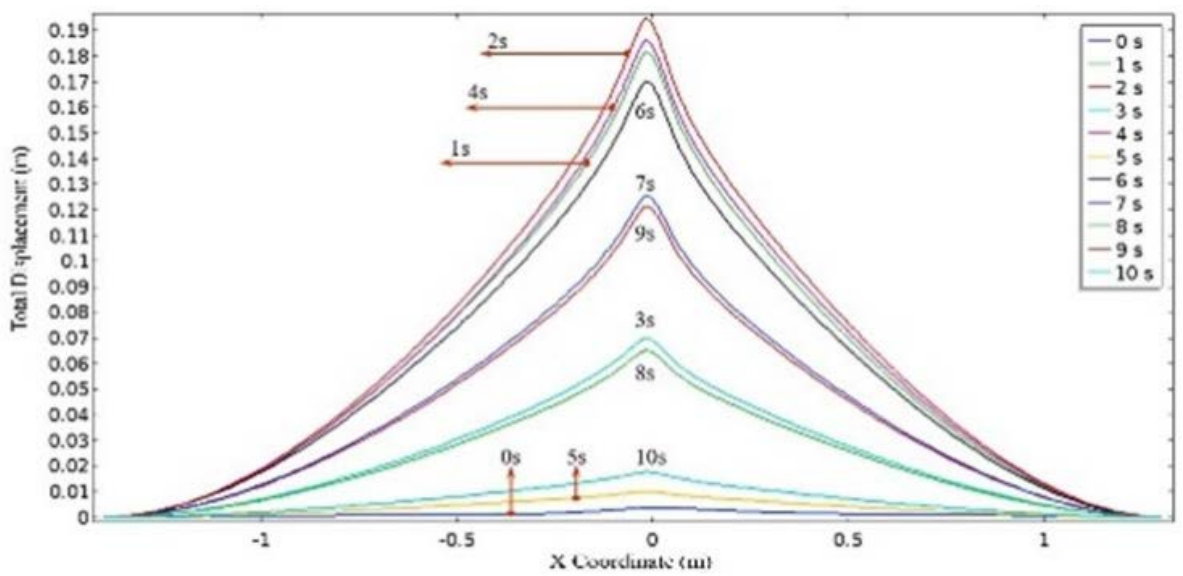

(b)

Figure 4: Monitoring point time - displacement curve in Parallel Basin.

Under the action of verticality basin, the time-history displacement of monitoring point B1 in different directions shows the opposite law to parallel basin, which is mainly dominated by displacement in $\mathrm{z}$ direction, and the $\mathrm{x}$, $\mathrm{y}$ direction still maintains the trend of symmetric fluctuation, and the amplitude is low. The transport pipeline is fixed in the verticality basin. Therefore, due to the velocity vector direction of the tidal flow and the mechanical properties of the solid structure of the pipeline itself, the time-history displacement in the $\mathrm{x}$, y direction is mainly implied by the fluctuation of the $\mathrm{z}$ direction and the $\mathrm{x}, \mathrm{y}$ direction component of the fluid tidal flow of the basin. By calculating the time-history displacement of all monitoring points, the time-history displacement fluctuations of each monitoring point in the verticality basin are the same as those in the parallel basin and are consistent with the tidal flow period. The monitoring point B1 of the riser pipe section has a large displacement, and the single-pass amplitude reaches $0.55 \mathrm{~m}$. 
The displacement at the curved pipe is higher than the monitoring point at the entrance and exit, and the displacement fluctuation shows a uniform downward trend, which is different from the parallel flow field. The value of the single point of the monitoring point 1 is $0.25 \mathrm{~m}$. The calculation and analysis of the time-history displacement of the S-type pipeline axis is shown in Fig. 5(b). The fluctuation law of the time-history displacement curve at the axis of the transport pipeline in the verticality basin is similar to that of the parallel basin and is higher at $x=0$ and its vicinity. The time-history displacement curve is different from the axis in the parallel basin. Under this basin calculation condition, there is no sudden increase, and the time-history displacement at the axis is relatively gentle.

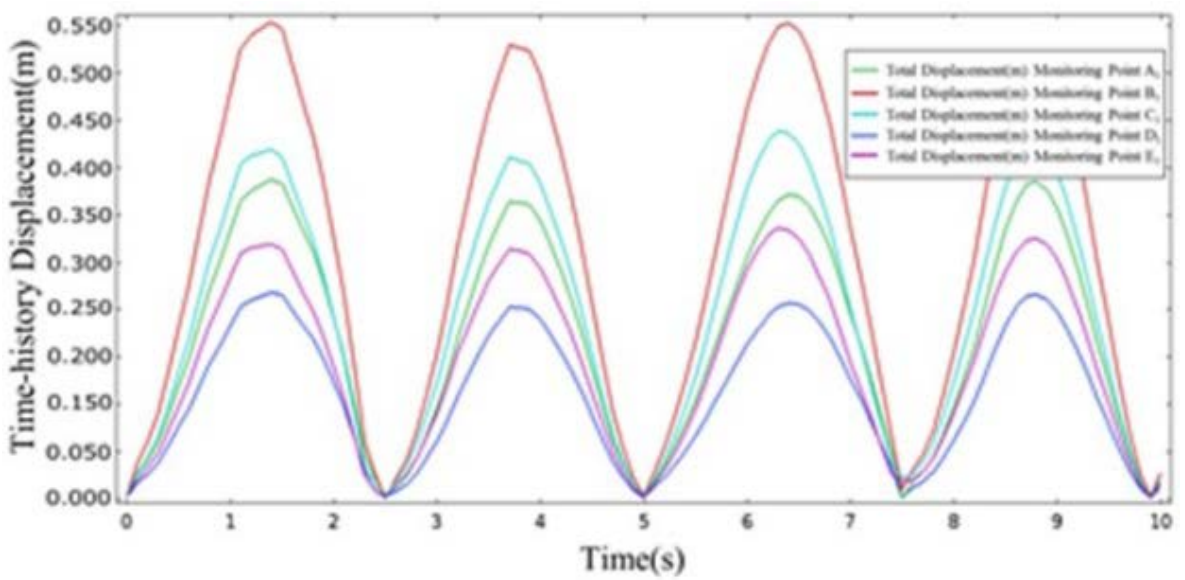

(a)

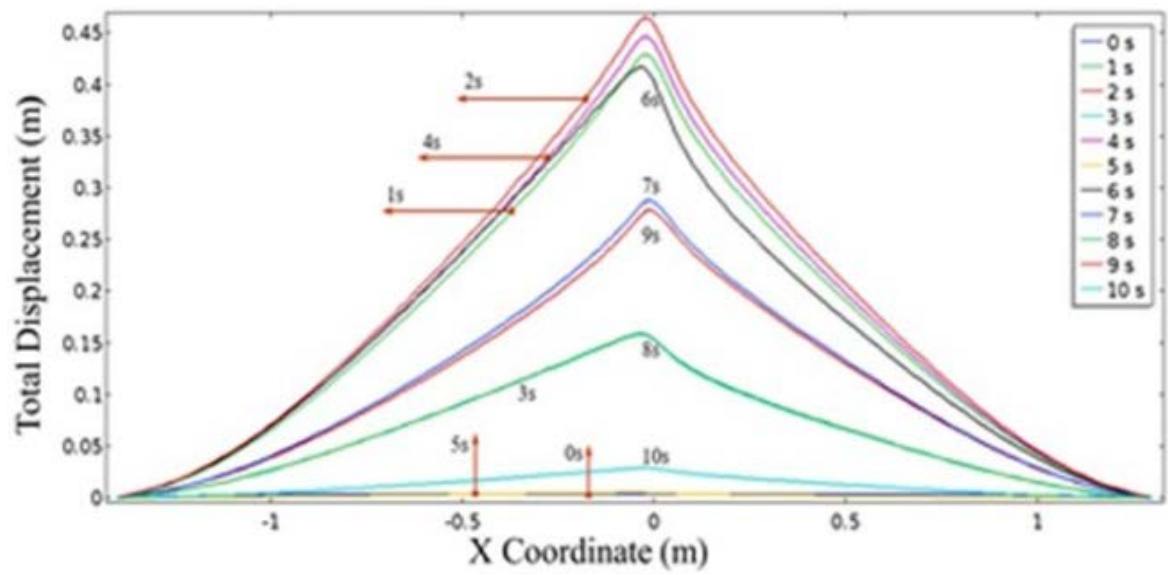

(b)

Figure 5: Monitoring point time - displacement curve in Verticality Basin 


\section{CONCLUSION}

Under the action of tidal flow in the parallel basin, the velocity field distribution of the outer basin shows a cyclical change law. The momentum transfer of the fluid through the pipeline leads to a lower velocity field on the deformation side. The stress of the transport pipeline under the outer basin manifests that the solid support end is higher than the riser area, which is higher than the curved pipeline area; the time-history displacement of the different monitoring points of the transport pipeline exhibits a periodic fluctuation law, and the singlepass displacement extreme value is $0.33 \mathrm{D}$, which is dominated by the displacement in the $\mathrm{x}$, $\mathrm{y}$ direction, and the displacement magnitude in the $\mathrm{z}$ direction is relatively lower, the singlepath displacement minimum value is $0.2 \mathrm{D}$; the pipe axis displacement suddenly increases in the riser pipe section, exhibiting a normal distribution, and an extreme value occurs at the center of the pipe section.

Under the tidal flow cycle of the verticality basin, the velocity field on the deformation side of the pipeline is low, and the low velocity region changes with the physical shape of the pipeline and its deformation vibration. The stress distribution of the pipeline wall is consistent with that of the parallel basin, and the solid support is high. The riser area is higher than the curved pipeline area, the pipeline generates symmetric vibration centered on its axis, the deformation of the pipeline is dominated by the $\mathrm{z}$ direction, and the time-history displacement components in the $\mathrm{x}, \mathrm{y}$ direction are of a lower order and symmetric distribution. The displacement is generally higher than that of the parallel basin. The time-history displacement is $1 \mathrm{D}$ and the minimum value is $0.5 \mathrm{D}$. The time-history displacement of the pipeline is relatively flat in the riser section.

\section{ACKNOWLEDGEMENT}

This research was funded by Natural Science Foundation of Heilongjiang Province: LH2019E017 Study on the non-steady Heat-seepage Coupling Law with Moving Boundaries of SW-SAGD with between Heel and Toe Injection in heavy oil reservoirs and University Nursing Program for Young Scholars with Creative Talents in Heilongjiang Province (No. UNPYSCT-2017035).

\section{REFERENCES}

[1] Paidoussism P, Lig X, Pipes conveying fluid: a model dynamical problem [J]. Journal of Fluid and Structures, 7: 137-204, 1993.

[2] Kuhnen J, Song B, Scarselli Dl, Destabilizing turbulence in pipe flow [J]. Nature Physics, 2018.

[3] Meng Dan, Guo Haiyan, Xu Sipeng, Stability analysis on flow-induced vibation of fluidconveying pipes [J]. Journal of Vibration and Shock, 29(6):80-83, 2010.

[4] Liang Feng, Bao Redong, Jin Ying, Nonlinear vibration of a fluid-conveying pipe under external excitation accompanied with internal resonance [J]. Journal of Vibration and Shock, (22), 2014.

[5] Leonardo Chica, Raresh Pascali, Paul Jukes et al. Jumper Analysis with Interacting Internal Two-Phase Flow[C]. Proceedings of the 22nd International Offshore and Polar Engineering Conference. Rhodes, Greece: ISOPE, 2012: ISOPE

[6] D. Jia. Slug Flow Induced Vibration in a Pipeline Span, a Jumper, and a Riser Section [C]. Offshore Technology Conference. Houston, Texas: OTC, 2012: OTC 22935. 
[8] Juan P. Pontaza, Raghu G. Menon. Flow-Induced Vibrations of Subsea Jumpers due to Internal Multi-phase Flow[C]. Proceedings of the ASME 30th International Conference on Ocean, Offshore and Arctic Engineering. Rotterdam, The Netherlands: OMAE, 2011: OMAE 50062.

[9] Ratkovich, N., Majumder, S., Bentzen, T.R., 2012. Empirical correlations and CFD simulations of vertical two-phase gas-liquid (Newtonian and non-Newtonian) slug flow compared against experimental data of void fraction. Chem. Eng. Res. Des. 91 (6), 988998.

[10] Gayet, C., N’Diaye, M., Liné, A., 2013. Behaviour of slug flow and pressure force induced in a spool: Numerical simulation of a taylor bubble flowing in a liquid flow through a spool. In: 16th International Conference on Multiphase Production Technology. BHR Group.

[11] Doussis M P P. The canonical problem of the fluid-conveying pipe and radiation of the knowledge gained to other dynamics problems across Applied Mechanics [J]. Journal of Sound \& Vibration, 2008,310 (3):462-492.

[12] Yong G, Xie J, Lin W, Three-dimensional vibration of cantilevered fluid-conveying micropipes-Types of periodic motions and small-scale effect [J]. International Journal of Non-Linear Mechanics, 102:112-135, 2018.

[13] Liu M, Wang Z, Zhou Z, Vibration response of multi-span fluid-conveying pipe with multiple accessories under complex boundary conditions[J]. European Journal of Mechanics - A/Solids, 72: S0997753817303856, 2018.

[14] Hong-Jun, Zhao, Hong-Lei., Experimental Investigation of Vibration Response of A Free-Hanging Flexible Riser Induced by Internal Gas-Liquid Slug Flow [J]. China Ocean Engineering, (6):633-645, 2018.

[15] Yang W, Ai Z, Zhang X, Nonlinear dynamics of three-dimensional vortex-induced vibration prediction model for a flexible fluid-conveying pipe [J]. International Journal of Mechanical Sciences, 138, 2018.

[16] Errico F, Ichchou M, Rose S D, The modelling of the flow-induced vibrations of periodic flat and axial-symmetric structures with a wave-based method[J]. Journal of Sound \& Vibration, 424:32-47, 2018.

[17] Ji Chunning, Chen Weilin, Numerical investigation on flow-induced vibration of two cylinders in tandem arrangements and its coupling mechanisms [J]. Chinese Journal of Theoretical and Applied Mechanics, 46(6):862-870, 2014.

[18] Chen Weilin, Ji Chunning, Numerical investigation on the asymmetric vibration and symmetry hysteresis of flow-induced vibration of two side-by-side cylinders [J]. Chinese Journal of Theoretical and Applied Mechanics, 47(5):731-739, 2015. 
248 